\title{
A SAÚDE MENTAL E ATENÇÃO PSICOSSOCIAL: \\ REGIONALIZAÇÃO E GESTÃO DO CUIDADO INTEGRAL NO SUS
}

MENTAL HEALTH AND PSYCHOSOCIAL CARE:

REGIONALIZATION AND MANAGEMENT OF INTEGRAL ON SUS

Recibido: 21 de septiembre de 2017 | Aceptado: 11 de enero de 2018

DOI: 10.22199/507187475.2018.0001.00004

\section{MAGDA DIMENSTEIN 1 ; JOÃO PAULO MACEDO 2 ; MAYARA GOMES 2 ; \\ TATIANE MENESES DA SILVA 2; MARIANA MARINHO DE ABREU 2}

1. UNIVERSIDADE FEDERAL DO RIO GRANDE DO NORTE, Programa de Pós-Graduação em Psicologia. Natal, RN, Brasil;

2. UNIVERSIDADE FEDERAL DO PIAUÍ, Departamento de Psicologia, Parnaíba, PI, Brasil. E-mail:

\section{RESUMO}

A Política de Saúde no Brasil tem avançado nos últimos anos em termos da regionalização da rede de serviços e da gestão do cuidado integral. No âmbito da saúde mental, a Rede de Atenção Psicossocial enfrenta obstáculos na sua implementação. Há localidades que não contam com a oferta adequada de serviços tanto no âmbito da atenção primária quanto no nível especializado. Deste modo, objetivou-se investigar as ações de saúde mental realizadas por equipes de atenção primária nesses vazios assistenciais. Realizou-se estudo de corte transversal, a partir dos dados de domínio público sobre a Rede de Atenção Psicossocial e das informações da avaliação do Programa Nacional de Melhoria do Acesso e da Qualidade da Atenção Básica (PMAQ). Foram identificadas fragilidades na articulação entre saúde mental e atenção primária em relação à longitudinalidade e coordenação do cuidado, à formação de vínculos, à orientação do trabalho para a comunidade e famílias e à competência cultural das equipes. À luz da Ontologia Política do Território compreende-se que as dificuldades relacionadas à regionalização e gestão do cuidado integral em saúde mental no Sistema Único de Saúde estão associadas à fragilidade do trabalho comunitário e territorial em saúde, que pressupõe o conhecimento do cotidiano, dos modos de vida e dos espaços por onde circulam os diferentes grupos sociais.

PALAVRAS-CHAVE: Atenção primária; saúde mental; regionalização; redes de atenção.

\begin{abstract}
Introduction: Health policy in Brazil has advanced considerably in terms of regionalization of network services and integral care management. Nevertheless, regarding mental health, the psychosocial care network faces implementation problems. Some places do not have adequate services both in specialized care and primary care. Objective: To analyze the mental health actions enacted by the primary care teams in these assistance gaps. Method: A cross-sectional study was carried out. Data were collected from public domain sites on psychosocial care network and the information on the National Program of Basic Care Access and Quality Improvement [Programa Nacional de Melhoria do Acesso e da Qualidade da Atenção Básica] (PMAQ). Results: Weaknesses were identified in the articulation between mental health and primary care in relation to the coordination of care, as well as the formation of bonds and community, family and cultural competence orientation. Conclusions: From the Ontological Territory Policy perspective, it is understood that the difficulties related to regionalization and management of mental health integral care in the SUS are associated to the weaknesses of community and territorial care which demands the knowledge of everyday life and the spaces where different social groups circulate.
\end{abstract}

KEY WORDS: Primary care; mental health; regionalization; care networks.

Aaradecimentos: Ao apoio recebido da Fundasão de Amparo à Pesquisa do Estado de São Paulo (FAPESP) processo número 2014/00701-1.

Endereço para correspondência com o Editor: Iara Falleiros Braga. E-mail: magda@ufrnet.br 
INTRODUÇÃO

No campo das políticas públicas, em especial no Sistema Único de Saúde (SUS), são cada vez mais evidentes os impasses em termos da melhoria do seu desempenho enquanto sistema e da consolidação da Atenção Primária (AP) como coordenadora de uma resposta integrada frente aos desafios atuais do perfil de morbimortalidade no país. Temos hoje o imperativo de construir uma nova institucionalidade para o SUS por meio da articulação das forças capazes de enfrentar o empobrecimento do seu devir existencializante, de resgatar seu caráter público, comprometido com a confrontação das desigualdades e injustiça social, capaz de abarcar a diversidade, a heterogênese maquínica, como diz Guattari (1992), ou seja, processos contínuos de singularização e criação na relação do ser humano com 0 mundo.

Nesse artigo, focaremos no processo de regionalização da saúde em curso no Brasil e de gestão e coordenação integral do cuidado nas Redes de Saúde, que é o cerne da modelagem necessária para uma atenção à saúde que seja pública, universal, equânime, integral e de qualidade em todo 0 território nacional. Partimos de duas situações como provocadoras dessa discussão:

1. Muitas das regiões de saúde do país são incapazes de atender $95 \%$ das necessidades de saúde da população. Os principais atributos da AP como a criação de vínculo, acompanhamento longitudinal, coordenação da atenção, integralidade, territorialidade e orientação comunitária, não são contemplados (Lima et.al., 2016);

2. Apesar da indicação pelo Ministério da Saúde de que atingimos $86 \%$ de cobertura no país em Saúde Mental, com a oferta de serviços extra hospitalares, continuam existindo vazios assistenciais importantes, falta de integração entre os pontos de atenção em um mesmo município e região de saúde, bem como um descompasso entre a oferta de cuidados e as necessidades em saúde mental das populações, especialmente daquelas que vivem fora dos grandes centros urbanos. Por vazios assistenciais denominamos certas regiões do país que não contam com nenhum ponto da Rede de Atenção da Rede de Atenção Psicossocial/RAPS, com exceção de alguns dispositivos da $\mathrm{AP}$, ou possuem uma estrutura não condizente com o padrão mínimo esperado em termos de cobertura nos territórios de saúde (Brasil, 2015).

Nesse sentido, objetivamos problematizar duas situações: a) 0 processo de regionalização em saúde e b) a gestão do cuidado integral no SUS e suas repercussões na saúde mental. Especificamente, propomos analisar como a RAPS está organizada no cenário da regionalização da saúde e a articulação entre Atenção Primária e Saúde Mental sob a perspectiva territorial.

O intuito é discorrer primeiramente sobre o processo de regionalização da política de saúde no Brasil e argumentar que os problemas vivenciados na efetivação das redes de saúde, dentre elas a RAPS, e no cumprimento dos atributos da AP em todo território nacional, superam o âmbito das determinações macroestruturais relacionadas à fragmentação das políticas e do sistema de saúde, ao sub financiamento, à gestão pública, à politicagem, ao clientelismo, etc. Em seguida, pretendemos discutir, com base no antropólogo colombiano Escobar (2014), que as situações acima destacadas estão articuladas às dinâmicas territoriais $e$ comunitárias de muitos grupos sociais que vêm sofrendo erosão sistemática da sua base ontológica-territorial, particularmente daqueles que primam por concepções de mundo não dualistas, não baseadas nas separações humano/não humano, 
indivíduo/sociedade,

natureza/cultura, mente/corpo, razão/emoção. secular/sagrado,

\section{METODO}

Trata-se de um estudo de corte transversal, realizado em duas etapas. $\mathrm{Na}$ primeira utilizamos os dados disponibilizados em domínio público pela Coordenação Nacional de Saúde Mental e pelo Departamento de Atenção Básica (DAB) do Ministério da Saúde. As informações foram coletadas nas respectivas bases de dados em janeiro de 2016, de maneira a compor um quadro geral dos serviços que integram os principais pontos de atenção da RAPS no âmbito do SUS: atenção primária, atenção psicossocial especializada, atenção residencial de caráter transitório, atenção hospitalar e estratégias de desinstitucionalização.

$\mathrm{Na}$ segunda etapa focamos na base de dados, também de domínio público, da Avaliação Externa do Programa Nacional de Melhoria do Acesso e da Qualidade da Atenção Básica (PMAQ), concernentes ao primeiro ciclo da avaliação. São informações sobre o trabalho da equipe, organização do serviço, as ações ofertadas e 0 acesso e satisfação da populaçãousuária. Demos destaque aos itens relacionados às ações de saúde mental na AP.

$\mathrm{Na}$ análise das duas etapas contamos com 0 auxílio do software Statistical Package for the Social Sciences (SPSS) para identificar o parque de serviços da RAPS de forma regionalizada; e caracterizar as equipes e estabelecer as frequências de cada item investigado no PMAQ associado à saúde mental em relação aos atributos que caracterizam a AP: primeiro contato, longitudinalidade, abrangência / integralidade, coordenação, orientação para a comunidade, centralidade na família e competência cultural (Starfield, 2002). Tal estratégia metodológica serviu para dimensionar 0 processo de Reforma
Psiquiátrica no tocante à oferta de serviços, bem como aos vazios assistenciais em determinados territórios no país.

\section{RESULTADOS E DISCUSSÃO}

\section{1) Regionalização da Saúde - do que se trata e quais problemas enfrenta?}

A Regionalização da Política de Saúde no Brasil é uma estratégia para garantir a organização e oferta dos serviços, acesso, eficiência, redução de custos, aumentar satisfação do usuário, diminuir desigualdades, produzir impactos positivos nas condições sanitárias e na vida das populações. Objetiva superar a fragmentação da oferta de serviços de saúde por meio da ação cooperativa entre municípios, respeitando a diversidade dos contextos regionais, suas diferenças socioeconômicas e necessidades de saúde da população (Santos \& Campos, 2015).

As Regiões de Saúde foram definidas a partir de um recorte territorial ou espaço geográfico contínuo, constituído por agrupamentos de municípios limítrofes, com características culturais, econômicas e sociais semelhantes e redes de comunicação e infraestrutura de transportes compartilhados (Brasil, 2010). As Redes de Atenção à Saúde (RAS) são arranjos organizativos de ações e serviços de saúde de diferentes densidades tecnológicas e níveis de complexidade do sistema SUS, que integradas por meio de sistemas de apoio técnico, logístico e de gestão, buscam garantir a integralidade do cuidado (Brasil, 2010). Foram estabelecidas cinco redes temáticas de atenção no país, dentre elas a Rede de Atenção Psicossocial (RAPS), com prioridade para o enfrentamento do álcool, crack, e outras drogas.

Esse recente processo de regionalização da saúde no país enfrenta inúmeros obstáculos e tem sido alvo de muita discussão (Almeida et al., 2016, Lima, 2015, Ribeiro, 2015, Santos \& Campos, 2015). Em debate promovido pela Fiocruz sobre os 
"Desafios da Regionalização e Conformação de Redes de Atenção em Contexto de Desigualdades Territoriais ${ }^{1 ",}$ os pontos problemáticos apontados pelos pesquisadores dizem respeito à enorme desigualdade das condições socioeconômicas e à diversidade territorial nas diferentes regiões. Em função disso, observa-se algumas particularidades em relação aos arranjos populacionais, bem como em termos do perfil de morbimortalidade que não podem ser desconsiderados quando se planeja a cobertura em saúde de forma regionalizada e equitativa.

Essa desigualdade de condições das regiões não está descolada também das desigualdades observadas quanto à oferta de serviços, de infraestrutura física e de recursos humanos qualificados nas mesmas. Esses aspectos impactam, consequentemente, na quantidade $e$ qualidade do acesso e utilização dos recursos de saúde, na capacidade resolutiva das equipes, no cuidado continuado e na atuação no território. Os pesquisadores chamam atenção que há igualmente uma grande variedade entre as regiões no que diz respeito às necessidades de saúde das populações, a qual está articulada ao perfil de morbimortalidade, ao dinamismo demográfico e às formas de ocupação dos territórios.

Sabendo-se que $89 \%$ dos municípios do país são de pequeno porte (IBGE, 2010), é possível considerar que os problemas se complexificam quando pensamos em disponibilidade de recursos físicos e humanos, em oferta de serviços, em ações intersetoriais, em comparação às metrópoles. Nesse sentido, a lógica de organização do sistema de saúde necessita de dinamismo e flexibilidade na oferta de cuidados múltiplos e simultâneos, articulados às características dos territórios e da população, aspectos que não vêm sendo contemplados.
Outro ponto problemático para a configuração de redes integradas e regionalizadas refere-se ao subfinanciamento do setor público, à forte dependência do setor privado, à dificuldade de colaboração entre os municípios que estão sempre em conflito e competição, à multiplicidade de instâncias responsáveis pela coordenação. Por fim, observa-se a predominância da racionalidade instrumental/técnica e a fragilidade dos mecanismos de regulação do trabalho em saúde, aspectos que são históricos desde a implantação do SUS.

Quanto ao processo de regionalização da RAPS, de acordo com o Ministério da Saúde (Brasil, 2015), a ampla cobertura alcançada, com $86 \%$ de serviços extra hospitalares, resultou em um maior número e diversidade de serviços implantados nas regiões do país, tendência à interiorização da assistência em saúde mental para municípios de médio e pequeno portes, crescente participação na $\mathrm{AP}$, descentralização dos leitos de atenção psicossocial e Serviços Residenciais Terapêuticos das grandes para cidades menores.

Porém, apesar desse incremento considerável na efetivação de uma rede integrada continuam existindo uma estrutura não condizente de serviços, respeitando 0 padrão mínimo esperado em termos de cobertura. Além disso, o problema não se restringe apenas à baixa cobertura em muitas áreas, mas, especialmente, à falta de integração dos pontos de atenção em um mesmo município e, principalmente, em uma mesma região de saúde. Para complicar o cenário, se não bastasse a baixa cobertura e a falta de integração entre os serviços, há um inequívoco descompasso entre o que é ofertado e o que é demandado pela população, particularmente por quem vive fora dos grandes centros urbanos.

Sem desconsiderar a importância da regionalização para que as redes de atenção à saúde sejam sistêmicas e resolutivas, e 
que autores como Santos e Campos (2015) "concluam que o único caminho para o SUS ser nacional é regionalizá-lo e dotar a região de saúde de todo o instrumental necessário à gestão compartilhada, interfederativa e responsável" (p.438), dificuldades de acesso, segmentação entre serviços assistenciais e fragmentação no cuidado ainda podem ser identificadas. Assim, a regionalização da saúde é um ponto absolutamente desafiador na conjuntura atual.

\section{2) A RAPS e o cenário da saúde mental}

A RAPS fundamenta-se no paradigma da Atenção Psicossocial. Sua meta é garantir acesso aos cuidados integrais com qualidade, organizar os serviços em rede com 0 estabelecimento de ações intersetoriais e continuidade do cuidado, desenvolver ações com ênfase em serviços de base territorial e comunitária (Brasil, 2011a). Em termos organizativos, os dados encontrados sinalizam que 0 ordenamento da Atenção Psicossocial se estrutura em diferentes pontos da rede SUS, desde a AP, passando pela atenção psicossocial especializada, residencial, hospitalar, até às estratégias de desinstitucionalização.

O avanço na implantação de serviços da RAPS a partir de 2011 é uma realidade. Registra-se a ampliação dos pontos de acesso em saúde mental e a diversidade de serviços implantados nas regiões do país. Nota-se tendência à interiorização da assistência para municípios de médio e pequeno portes, com crescente participação da AP e descentralização dos leitos de atenção psicossocial e Serviços Residenciais Terapêuticos. Isso tem sido possível devido às mudanças na política de financiamento ao priorizar ações extra hospitalares e de base territorial e comunitária, além das pactuações nas três esferas de governo e níveis de governança regional, com vistas a estruturar uma ampla rede de serviços psicossociais no país.
Inclui-se nesse processo alguns desafios, especialmente relacionados à cobertura em certas regiões do país, com locais sem oferta de infraestrutura ou de recursos humanos conforme padrões preconizados pelas políticas públicas de saúde. Apesar da ampliação da taxa de crescimento e cobertura em todo o país, em termos da distribuição de serviços do tipo Centro de Atenção Psicossocial (CAPS), Serviço Residencial Terapêutico (SRT), Unidades de Acolhimento (UA) e leitos de saúde mental em hospital geral, há diferenças regionais importantes que trataremos a seguir.

Para CAPS e SRT, o Centro Oeste e Norte são as regiões que apresentam piores índices. Quando visualizamos a distribuição da atenção psicossocial especializada a partir do modelo de agrupamento da Comissão Intergestora Regional (CIR) proposto por Viana et al. (2015) é possível perceber iniquidades na oferta desses serviços. Nas regiões de saúde caracterizadas por baixo desenvolvimento socioeconômico e baixa oferta de serviços, há menor cobertura de CAPS 24 horas e CAPS Álcool/Drogas (AD) 24horas, inclusive nas localidades com porte populacional que justificam sua implantação. Situação mais delicada ainda é a do CAPS II, CAPS AD e CAPS Infantil, pois apesar de serem serviços que podem ser implantados em localidades com menor porte populacional (acima de 70 mil hab.) estão localizados em maior percentual nas regiões de saúde mais desenvolvidas.

Em relação à $A P$, apesar da ampla cobertura no país, observamos situações de vazios assistenciais neste nível de atenção com muita preocupação. Em relação ao Núcleo de Apoio à Saúde da Família (NASF), pelo menos 1.798 municípios do país não contam com este equipamento, sendo 0 destaque para as localidades das regiões menos desenvolvidas. No caso da Estratégia Saúde da Família (ESF), são 31 municípios que não contam com equipes em suas localidades, com destaque para as regiões 
com alta oferta de serviços. O mesmo ocorre com os Agentes Comunitários de Saúde (ACS), que são 17 municípios que se encontram nesta mesma condição.

É evidente que a carência de serviços especializados, e principalmente de AP nas regiões de saúde, configura-se como um ponto crítico e de parada no processo de expansão e regionalização da RAPS no país para responder de forma substitutiva às necessidades de internação em hospitais psiquiátricos, mas também aos casos de transtornos psiquiátricos menores, pacientes crônicos, gestão da crise no território, comportamento suicida, assistência ao público infantil (autismo e medicalização do sujeito escolar), necessidades decorrentes do uso do crack, do álcool e outras drogas, suporte familiar, violência social, etc. (Vasconcelos, 2010). Portanto, é preciso expandir a cobertura assistencial em saúde mental em todo o território nacional e superar os vazios assistenciais.

Atualmente existem 13 Regiões de Saúde dentre as 438 existentes em todo o país nesta situação crítica, o que corresponde a 104 municípios que têm apenas um ponto de atenção da RAPS (serviços com contam com equipes de ACS, ESF e/ou NASF). Trata-se de um quadro que sinaliza fragilidade da Política Brasileira de Saúde Mental e a dificuldade no acesso e qualidade da atenção psicossocial nestas localidades, resultando em peregrinações dos usuários e familiares, oriundos de municípios com baixa oferta de serviços, em direção às capitais e/ou grandes centros urbanos, em busca de atendimento especializado.

Essas 13 Regiões de Saúde se destacam pelo baixo nível de desenvolvimento socioeconômico e baixa oferta de serviços ( $\mathrm{n}$ = 6) e, contraditoriamente, com médio nível de desenvolvimento socioeconômico e alta oferta de serviços $(n=5)$. É possível observar que a população residente nos municípios dessas Regiões de Saúde soma 1.677.550 habitantes, que contam somente com equipes da atenção primária para responder às necessidades em saúde mental. Além da baixa cobertura, falta integração entre os pontos de atenção no município e na Região de Saúde. Ou seja, apesar da importância e benefícios das redes regionalizadas e integradas de atenção à saúde, dificuldades de acesso, segmentação entre serviços assistenciais e fragmentação no cuidado ainda podem ser identificadas. Isso se aplica mais fortemente às populações rurais, assentadas e quilombolas (Lima et. al., 2016).

Concluímos, nesta primeira etapa, que o processo de regionalização da RAPS encontra-se ainda muito incipiente no país. Temos um quadro de distribuição desigual de equipes e serviços, dificuldades no processo de pactuação entre os municípios, barreiras de acesso e fraca articulação com a AP. Vivemos um cenário onde os agravos decorrentes de transtornos mentais severos estão cada vez mais evidentes, associados às doenças crônicas em geral, uso excessivo de álcool e outras drogas e condições precárias de vida. $O$ fracasso das respostas na perspectiva psicossocial e a persistência do hospital psiquiátrico são evidentes. A aposta no modelo de atenção comunitária, interdisciplinar e continuada que caracteriza a proposta dos cuidados primários em saúde parece longe de estar consolidado enquanto paradigma de trabalho no SUS. A seguir, vamos destacar alguns atributos da AP avaliados pelo PMAQ que se destacaram como mais problemáticos em relação à saúde mental e ao cuidado psicossocial.

\section{3) A saúde mental e o cuidado psicossocial avaliados pelo $P M A Q$}

Desde de 2011, a qualidade da atenção e da gestão da AP assumiu maior relevância. Algumas ações de aprimoramento para qualificar sua capacidade de resposta às necessidades de saúde da população foram implementadas pelo Ministério da Saúde, dentre elas a criação do PMAQ em 2011 (Brasil, 2011b). 
Foram 17.482 equipes pesquisadas no primeiro ciclo, assim distribuídas: Sudeste $(38,2 \%)$, Nordeste $(32,3 \%)$, Sul $(17 \%)$, Centro-oeste $(6,4 \%)$ e Norte $(6,1 \%)$. A maioria está localizada em municípios no interior do país $(83 \%)$, principalmente de pequeno porte (52\%). Em relação à modalidade das equipes e profissionais que compõem a Atenção Básica de todo o Brasil, a maior parte é formada por equipes de Saúde da Família (médico, enfermeiro, auxiliar/técnico em enfermagem e agentes comunitários de saúde) com a presença de profissionais de saúde bucal.

De acordo com o DAB, em 2013, a Atenção Básica contava com 323.270 Agentes Comunitários de Saúde e 44.253 Equipes de ESF, o que correspondia, respectivamente, a $64,74 \%$ e $56,37 \%$ de cobertura populacional. Para Malta et al. (2016), o principal desafio, além da necessidade de ampliar a cobertura, está na dificuldade de fixar profissionais, especialmente os médicos, devido à grande rotatividade, em especial em cidades do interior e nas periferias dos grandes centros urbanos.

Nesse estudo, damos especial atenção, dentre as equipes de saúde de todo o país investigadas no $P M A Q$, àquelas inseridas nas áreas que não contam com equipamentos especializados da RAPS em seu território (vazios assistenciais). Deste modo, centramo-nos nas 157 equipes que atuam em 70 municípios, pertencentes às 13 Regiões de Saúde referidas no tópico anterior. Estão situadas nos estados brasileiros do Sudeste $(75,2 \%)$, com destaque para São Paulo (74 equipes) e Minas Gerais (44 equipes), seguido do Centro-oeste (14\%) e no Norte $(10,8 \%)$. Essas 157 equipes estão localizadas, sem exceção, em munícipios do interior e predominantemente de pequeno porte (79\%), dado que sinaliza a dificuldade da interiorização e regionalização da assistência em saúde mental. A seguir, focaremos nos atributos da AP avaliados pelo $P M A Q$ que se destacaram como mais problemáticos em relação à saúde mental e ao cuidado psicossocial, inclusive nas áreas reconhecidas como de vazios assistenciais.

\section{1) Primeiro Contato - Acolhimento e organização da agenda em Saúde Mental}

Para autores como Starfield (2002), Giovanella e Mendonça (2012), o "primeiro contato" é um atributo definidor da AP como porta de entrada preferencial do sistema de saúde. O serviço deve constituir-se como acessível à população, eliminando possíveis barreiras (geográficas, organizacionais e culturais), no sentido de garantir 0 acesso à própria unidade de saúde ou à atenção especializada, por meio de encaminhamentos e coordenação do cuidado.

Lima et al. (2016), ao relacionarem esse atributo com as dimensões investigadas pelo instrumento da avaliação externa do PMAQ, consideraram as seguintes variáveis que indicam ações de primeiro contato: "horários de funcionamento", "acolhimento", "agendamento às consultas na Atenção Básica" e "atendimento de urgência e emergência". Dessas variáveis, identificamos somente duas como objeto de análise neste atributo relacionadas à saúde mental: 0 acolhimento e a organização da agenda. $O$ acolhimento é uma tecnologia relacional com 0 objetivo de rever necessidades e prioridades, realizar classificação por risco e evitar a espera desnecessária dos usuários. A organização da agenda deve contemplar a demanda espontânea e a programada, de modo a articular a integralidade das ações por meio de diretrizes clínicas e territoriais, garantindo a continuidade do cuidado.

Apresentamos na Tabela 1 os resultados das variáveis identificadas no atributo "primeiro contato". 
TABELA 1.

Respostas das equipes ESF de todo o país e em localidades com vazios assistenciais em Saúde Mental sobre as ações do atributo "primeiro contato".

\begin{tabular}{|c|c|c|}
\hline Variáveis & Geral & $\begin{array}{c}\text { Vazios } \\
\text { Assistenciais }\end{array}$ \\
\hline Acolhimento implantado & $80 \%$ & $81,5 \%$ \\
\hline $\begin{array}{l}\text { Dispõem de profissionais capacitados para avaliação, } \\
\text { classificação de risco e vulnerabilidade dos usuários }\end{array}$ & $46,3 \%$ & $38,2 \%$ \\
\hline $\begin{array}{l}\text { Atendem todo e qualquer usuário que chega à unidade de saúde } \\
\text { espontaneamente, escutando e avaliando suas necessidades. }\end{array}$ & $97,2 \%$ & $95,5 \%$ \\
\hline Atendem casos de saúde mental & $30,1 \%$ & $26,1 \%$ \\
\hline $\begin{array}{l}\text { Possuem agenda semanal de atividades pactuada pelo } \\
\text { conjunto de profissionais que integram a equipe }\end{array}$ & $81,2 \%$ & $67,5 \%$ \\
\hline $\begin{array}{l}\text { Programam consultas e ações para usuários que fazem parte de } \\
\text { programas ou grupos prioritários e necessitam de cuidado continuado }\end{array}$ & $91 \%$ & $85,4 \%$ \\
\hline Programam consultas e ações para usuários em saúde mental & $44,3 \%$ & $42 \%$ \\
\hline Agendamento de consultas para portadores de transtorno mental & $56 \%$ & $58,6 \%$ \\
\hline
\end{tabular}

Apesar do avanço na implantação do acolhimento nos serviços de AP em todo 0 país, as equipes não dispõem de profissionais capacitados para avaliação, classificação de risco e vulnerabilidade dos usuários. Por outro lado, mesmo as equipes atendendo mais de $95 \%$ dos usuários que chegam espontaneamente nos serviços, quando se trata de casos de saúde mental 0 percentual cai significativamente. Embora 0 percentual de ações de planejamento de consultas para grupos prioritários que necessitam de cuidado ter sido alto e 0 tempo de espera para a primeira consulta ser em média sete dias ( $d p=15,42)$, menos da metade das equipes oferecem de forma programada ações direcionadas para portadores de transtornos mentais.

As equipes localizadas nas áreas de vazios assistenciais apresentaram índices mais delicados do que as demais. Talvez, por contarem com menor retaguarda de serviços especializados de diferentes redes temáticas, bem como de outros níveis de atenção, as repostas relacionadas ao agendamento de consultas são um pouco melhores que as das equipes do resto do país, pois $58,6 \%$ o fazem em qualquer dia da semana e em qualquer horário, embora 0 tempo de espera tenha permanecido 0 mesmo.

No geral são dados que preocupam e fragilizam a capacidade de resposta da AP para os casos de saúde mental, quer em regiões que comportam em sua rede diversos pontos de atenção da RAPS, quer em localidades com reduzida presença de serviços. Faz-se necessário maior investimento quanto a adoção de ferramentas relacionais e de reorientação dos processos de trabalho a partir do planejamento das ações e 0 compartilhamento do processo decisório para potencializar o acolhimento dos casos de saúde mental, rompendo com possíveis barreiras organizativas, burocráticas e socioculturais que tanto restringem 0 acesso dos usuários aos serviços (Starfield, 2002; Giovanella \& Mendonça (2012).

3.2) Abrangência ou Integralidade - serviços realizados/escopo das ações e resolutividade da $A B$

Abrangência ou integralidade refere-se ao reconhecimento do conjunto ampliado de necessidades da saúde e suas determinações, por parte das equipes de saúde, com a oferta de serviços diversificados na perspectiva da produção e 
continuidade do cuidado (preventivos, promocionais e curativos). No caso da equipe não dispor de recursos para garantir a resolutividade do problema, deve assegurar 0 acesso a outros serviços de diferentes níveis de complexidade, por meio do encaminhamento e de maneira coresponsabilizada (Giovanella \& Mendonça, 2012, Starfield, 2002).

Lima et al. (2016) consideraram pelo menos duas variáveis investigadas no instrumento de avaliação externa do PMAQ que indicam ações de abrangência ou integralidade: "serviços realizados/escopo de ações" (consultas, procedimentos, etc) e "resolutividade da $A B$ " (disponibilidade de profissionais, suporte do NASF e outros serviços, ações de educação permanente, etc).

Sobre os resultados do atributo "abrangência ou integralidade" direcionados aos casos de saúde mental, estão organizados da seguinte maneira:

\section{TABELA 2.}

Respostas das equipes ESF de todo o país e em localidades com vazios assistenciais em Saúde Mental sobre as ações do atributo "abrangência ou integralidade".

\begin{tabular}{lcc}
\hline \multicolumn{1}{c}{ Variáveis } & Geral & $\begin{array}{c}\text { Vazios } \\
\text { Assistenciais }\end{array}$ \\
\hline $\begin{array}{l}\text { Realizam consultas específicas com tempo maior de } \\
\text { atendimento para os casos de saúde mental }\end{array}$ & $40,5 \%$ & $21,7 \%$ \\
$\begin{array}{l}\text { Utilizam o registro da história de vida como estratégia } \\
\text { específica para cuidar dos casos de saúde mental } \\
\text { Ofertam atendimento em grupo como estratégia específica } \\
\text { para cuidar dos casos de saúde mental }\end{array}$ & $42,7 \%$ & $24,8 \%$ \\
$\begin{array}{l}\text { Tiveram algum tipo de preparação para o atendimento de } \\
\text { usuários com transtorno mental }\end{array}$ & $26,4 \%$ & $11,5 \%$ \\
$\begin{array}{l}\text { Possuem registro do número dos casos mais graves de } \\
\text { usuarios com transtorno mental }\end{array}$ & $32,2 \%$ & $25,5 \%$ \\
$\begin{array}{l}\text { Possuem registro dos usuários com necessidades decorrente } \\
\text { do uso de crack, álcool e outras drogas }\end{array}$ & $58,4 \%$ & $39,5 \%$ \\
$\begin{array}{l}\text { Possuem registro dos usuários em uso crônico de benzodiazepínicos } \\
\text { Realizam ações para pessoas que fazem uso crônico de }\end{array}$ & $23,5 \%$ & $26,1 \%$ \\
$\begin{array}{l}\text { benzodiazepínicos e necessitam desmame (diminuição da dose) } \\
\text { Contam com ações de matriciamento na resolução de }\end{array}$ & $42,3 \%$ & $45,2 \%$ \\
$\begin{array}{l}\text { casos considerados complexos } \\
\text { Realizam reuniões para qualificação clínica com participação } \\
\text { de equipes de apoio matricial (NASF, CAPS, especialidades) }\end{array}$ & $22,1 \%$ & $21 \%$ \\
\hline
\end{tabular}

São dados bastante preocupantes, visto que tal cenário impacta sobremaneira na abrangência e resolutividade da AP em relação aos casos de saúde mental. Os resultados expõem a fragilidade da assistência ofertada, sobretudo pelas equipes não contarem com a devida preparação para 0 atendimento em saúde mental. Isto incide nas poucas equipes que registram casos em saúde mental no território, bem como no desdobramento das ações realizadas (consultas, acompanhamentos e atividades em grupo), integrando a perspectiva da promoção, da prevenção e da reabilitação psicossocial no próprio território.

Tais dificuldades ganham novos contornos quando constatamos que $88,5 \%$ das equipes do país contaram com ações de matriciamento na resolução de casos considerados complexos, pois suspeita-se que os casos de saúde mental podem não estar sendo contemplados nessas ações. Além disso, há o agravante que um pouco mais da metade das equipes não realiza 
reuniões para qualificação clínica com participação de equipes de apoio matricial (NASF, CAPS, especialidades). Tal fato repercute na fragilidade do suporte e pode indicar que as ações de matriciamento não têm avançado em termos de apoio técnicopedagógico, vínculo interpessoal e apoio institucional necessários no sentido de constituir-se como dispositivo de educação permanente para construção coletiva de projetos terapêuticos voltados para qualificar a atenção psicossocial na atenção primária (Chiaverini, 2011). Nas localidades consideradas vazios assistenciais os dados ganham proporções ainda mais assustadoras, especialmente pela pouca penetrabilidade das ações de saúde mental desenvolvidas pelas equipes da AP.

\section{3) Coordenação do Cuidado -} Ordenamento dos fluxos assistenciais

A atenção ininterrupta do cuidado é imprescindível para que a atenção integral seja garantida. Para tanto, envolve tecnologias de gestão clínica, mecanismos adequados de comunicação entre profissionais e registro adequado de informações. Deste modo, faz-se necessário dispor de informações do projeto terapêutico do caso atendido, do histórico dos problemas de saúde e do conjunto de necessidades do indivíduo e sua família (Giovanella \&
Mendonça; 2012, Starfield, 2002). Isso exige um prontuário de acompanhamento longitudinal (ao longo da vida) que oriente 0 encaminhamento para serviço ou nível mais especializado de atenção e que tais informações possam ser disponibilizadas. 0 caminho inverso das informações (do nível especializado para equipe da AP) é de suma importância, no objetivo do acompanhamento e fortalecimento do cuidado. A ação permanente de gestão dos processos de trabalho pelas equipes é fundamental para operar-se com a gestão da clínica no sentido de coordenar o sistema de referências/fluxos dos casos entre os serviços.

Com base em Lima et al. (2016), consideramos a seguintes variáveis presentes no instrumento de avaliação externa do PMAQ que indicam ações de coordenação do cuidado: "continuidade informacional", "ordenamento de fluxos assistenciais", "acesso à consulta/exames especializados" e "comunicação direta entre serviços da Atenção Básica e Especializada".

A Tabela 3 apresenta os resultados das variáveis identificadas no atributo "coordenação do cuidado".

\section{TABELA 3.}

Respostas das equipes ESF de todo o país e em localidades com vazios assistenciais em Saúde Mental sobre as ações do atributo "coordenação do cuidado".

\begin{tabular}{lcc}
\hline \multicolumn{1}{c}{ Variáveis } & Geral & $\begin{array}{c}\text { Vazios } \\
\text { Assistenciais }\end{array}$ \\
\hline $\begin{array}{l}\text { Realizam prontamente o agendamento para o paciente } \\
\text { quando é encaminhado para consulta especializada }\end{array}$ & $26,1 \%$ & $20,4 \%$ \\
$\begin{array}{l}\text { A consulta é marcada pela unidade de saúde e a data } \\
\text { posteriormente informada ao paciente }\end{array}$ & $61 \%$ & $64,3 \%$ \\
$\begin{array}{l}\text { Ao sair da unidade de saúde com consulta agendada o paciente recebe uma ficha } \\
\text { de encaminhamento/referência para o serviço ou profissional especializado }\end{array}$ & $34,6 \%$ & $45,9 \%$. \\
$\begin{array}{l}\text { Possuem protocolo com definição de diretrizes terapêuticas } \\
\text { para problemas relacionados à saúde mental }\end{array}$ & $43,3 \%$ & $28 \%$ \\
$\begin{array}{l}\text { Possuem protocolo com definição de diretrizes terapêuticas para problemas } \\
\text { relacionados a necessidades decorrente do uso de crack, álcool e outras drogas }\end{array}$ & $31,8 \%$ & $21,7 \%$
\end{tabular}


Os casos de saúde mental demandam, acima de tudo, diretrizes terapêuticas pautadas em ações de continuidade e coordenação de cuidados em articulação permanente com outros pontos de atenção das redes de saúde e intersetoriais. Não é o que acontece na grande maioria das equipes do país e em especial nas localidades com vazios assistenciais identificadas nesse estudo. Tais lacunas resultam em graves obstáculos quanto à interiorização e regionalização da assistência, bem como à integração da saúde mental ao SUS.

\section{4) Nivel de satisfação dos usuarios}

Participaram do PMAQ 65.391 usuários dos serviços, assim distribuídos nas regiões do país: Sudeste $(38,9 \%)$, Nordeste $(33 \%)$, Sul $(15,8 \%)$, Centro-Oeste $(6,6 \%)$ e Norte $(5,7 \%)$. Sendo que $82,8 \%$ vivem no interior, sobressaindo as localidades de pequeno porte populacional (53\%) e $17,2 \%$ nas capitais. Do total dos 19 itens pesquisados sobre o acesso, utilização, participação e satisfação do usuário, selecionamos dois atributos: "orientação para a comunidade" e "longitudinalidade/vínculo".

O atributo orientação para a comunidade trata do amplo conhecimento das necessidades da população residente em cada território que as equipes devem ter, envolvendo ainda 0 contexto socioeconômico e cultural da mesma, 0 mapa da situação de saúde do território, os equipamentos/serviços disponíveis e participação da comunidade. Sobre este atributo focamos uma variável específica do processo de territorialização, que trata da adequação das soluções propostas pelas equipes à realidade dos usuários. As respostas foram: sempre $(48,9 \%)$, nunca $(22,4 \%)$, na maioria das vezes (18\%), quase nunca $(7,2 \%)$ e não sabe/não respondeu $(3,4 \%)$. Nas localidades reconhecidas como vazios assistenciais em relação à RAPS, os índices de respostas foram: sempre $(61,9 \%)$, nunca $(21,8 \%)$, na maioria das vezes $(9,5 \%)$, quase nunca $(4,4 \%)$ e não sabe/não respondeu $(2,4 \%)$, apontando uma maior competência cultural das equipes.

O atributo longitudinalidade/vínculo trata da responsabilidade da equipe para com 0 usuário, da relação profissional-paciente e da qualidade do vínculo (Giovanella \& Mendonça, 2012, Starfield, 2002). Focamos no vínculo, o qual indica se o usuário se sente à vontade para falar com a equipe sobre suas preocupações, problemas sociais, familiares ou outras questões. Os resultados nas equipes do país foram: sempre $(54,7 \%)$, nunca $(23,9 \%)$, na maioria das vezes $(13,3 \%)$, quase nunca $(6,5 \%)$, e não sabe/não respondeu (1,5\%). Do mesmo modo, nas localidades que contam apenas com equipes ESF e NASF, os índices de respostas foram: sempre $(58,2 \%)$, nunca $(28,7 \%)$, na maioria das vezes $(7,6 \%)$, quase nunca $(5 \%)$ e não sabe/não respondeu $(0,5 \%)$. Ou seja, em localidades com menor retaguarda de serviços da RAPS, mesmo com todas as fragilidades apresentadas em relação à realização de ações de saúde mental, os usuários apresentam melhores índices de satisfação com a assistência recebida e indicam que suas necessidades são reconhecidas por profissionais aos quais se sentem acolhidos e vinculados.

Que reflexões chegamos dessa avaliação para a gestão integral do cuidado em saúde e o cuidado psicossocial?

A parceria entre saúde mental e atenção primária, mais forte aposta no sentido da garantia da integralidade, se apresentou neste estudo como frágil e pouco resolutiva. Há equipes instaladas predominantemente em cidades do interior e de pequeno porte, com pouca oferta de serviços e insuficiência de profissionais qualificados. Em relação ao acolhimento em saúde mental a tônica é a falta de capacitação para avaliar os casos; menos da metade das equipes organiza suas agendas incorporando atividades em saúde mental e as consultas não são planejadas de acordo com as necessidades dos usuários. Estes, por sua vez, revelam a falta de 
conhecimento dos profissionais da suas realidades e problemas e, consequentemente, a não adequação das respostas ofertadas e fragilidade dos vínculos. A maior parte das equipes desconhece os usuários com transtornos mentais, com problemas associados ao uso abusivo de álcool e outras drogas e consumo excessivo de benzodiazepínicos na sua área de abrangência. Isso revela equipes pouco conhecedoras e atuantes nos territórios. 0 matriciamento, apesar de referido, não é operacionalizado em mais de $50 \%$ das equipes, seja nas UBS, NASF, seja partindo dos CAPS. A participação das diversas categorias profissionais nesse processo é baixa. $\mathrm{O}$ agendamento e acompanhamento desses casos de saúde mental não acontecem, indicando a falta de um cuidado regular e continuado. Esse quadro se agrava nas áreas consideradas como vazios assistenciais.

Em resumo, se requer não apenas 0 aprimoramento de estratégias para a efetivação do funcionamento dos serviços em rede, mas adequá-los à diversidade territorial e suas peculiaridades populacionais, pois vários atributos tais como vínculo, longitudinalidade, abrangência, coordenação do cuidado, orientação para a comunidade, centralidade na família e competência cultural, que formam a base de um modelo assistencial que tem em seu centro os sujeitos, não estão garantidos. Mas, por quê?

A razão encontra-se no fato que a gestão integral do cuidado implica em considerar os espaços de vida, conhecer as dinâmicas comunitárias, estabelecer vínculos, fazer um mergulho no território e na cotidianidade. Porque competência cultural dos profissionais implica em ser capaz de reconhecer as diferentes necessidades dos grupos populacionais em função de suas características étnicas, culturais e as diferentes concepções do processo saúdedoença-cuidado. Diz respeito à sensibilidade à diversidade e capacidade de reconhecer a inadequação e os limites das suas práticas e modelos interpretativos quando têm diante de si pessoas em sofrimento com outros códigos culturais e linguísticos.

A avaliação do PMAQ revelou muitos problemas nesse sentido, que os usuários não se sentem respeitados em relação aos seus hábitos culturais, costumes, religião, evidenciando desconhecimento e desqualificação das comunidades e territórios. Em razão disso, consideramos que os problemas relacionados à regionalização da saúde têm várias dimensões: política (relações de poder); econômica (financiamento e recursos), técnica (produção de conhecimentos e de tecnologias - saber fazer) e ontológica (visão de mundo e da existência humana). Em outras palavras, são efeitos da produção do saber, do poder e do ser.

Sabemos que a produção do conhecimento científico está fundamentada em uma ontologia dualista e universalista e em uma racionalidade instrumental que desconsidera a multiplicidade de formas de vida, a existência de diferentes mundos, a heterogeneidade espacial, social e simbólica que caracteriza os territórios, enfim, a ampla determinação dos processos de saúde, adoecimento, sofrimento e cuidado. As políticas públicas e as formações profissionais, por sua vez, estão geralmente ancoradas em uma ontologia orientada pelos valores da ciência: neutralidade, universalidade, hierarquia de conhecimentos, universo único, desenvolvimento e progresso. Narrativas universalistas e racionalidades instrumentais são produzidas com efeitos limitadores para os trabalhadores em saúde orientar suas ações profissionais com base na perspectiva da determinação social da saúde e do cuidado territorial, bem como para realizar ações interprofissionais sob a lógica do trabalho em equipe e em rede, visando à continuidade e longitudinalidade do cuidado. 
Chamamos atenção que desde o prisma das ontologias políticas relacionais, que prezam pela desnaturalização das concepções universalistas de mundo, jamais seremos bem-sucedidos na construção de redes e de uma gestão integrada de saúde se nos mantivermos ancorados na desvalorização, estigmatização e subordinação do conhecimento local, na hierarquização dos saberes, na separação do conhecer e do fazer.

A desqualificação das diferentes cosmovisões, 0 desconhecimento das relações e dinâmicas comunitárias, os essencialismos que não levam em consideração questões de gênero, étnicoraciais, intergeracionais, rurais e urbanas, todos esses aspectos constituem a base de sustentação dos saberes científicos e das maneiras de reproduzir o poder. 0 "devir existencializante" do SUS pressupõe, por sua vez, a inter-relação entre diferentes epistemes e rechaça a violência do colonialismo, do ocidentalismo, do eurocentrismo que oculta a persistente submissão e exploração das pessoas e da natureza.

Acreditamos que os problemas vivenciados na efetivação das redes de saúde, dentre elas a Rede de Atenção Psicossocial (RAPS) e o cumprimento dos atributos da AP em todo território nacional superam 0 âmbito das determinações macroestruturais. Ou seja, a formação de vínculo, o acolhimento, a continuidade de cuidados, a competência cultural dos profissionais, requerem operações nos domínios moleculares de sensibilidade, de inteligência e de desejo, tal como traz Guattari (1990) nas Três Ecologias, de maneira que precisa haver investimento de máquinas desejantes e criação de uma textura ontológica heterogênea que nos tire das incessantes re-territorializações conservadoras da subjetividade. Hoje, busca-se epistemologias alternativas. A expressão Epistemologias do Sul, cunhada pelo sociólogo Boaventura de Sousa Santos, é uma

"metáfora do sofrimento, da exclusão e do silenciamento de povos e culturas que ao longo da história foram dominados pelo capitalismo e colonialismo. Colonialismo que imprimiu uma dinâmica histórica de dominação política e cultural submetendo à sua visão etnocêntrica o conhecimento do mundo, o sentido da vida e das práticas sociais. Afirmação, afinal, de uma única ontologia, de uma epistemologia, de uma ética, de um modelo antropológico, de um pensamento único e sua imposição universal" (Tavares, 2009, p.183).

O colonialismo em suas formas contemporâneas de supressão e homogeneização dos saberes não está apenas nas relações globais norte-sul, ocidente-oriente, está no cotidiano, nas relações locais e particulares de saber e poder. É uma mentalidade e forma de sociabilidade autoritária e discriminatória. A imposição, 0 autoritarismo e não reconhecimento do saber do outro marcam as relações entre técnicos e usuários, homens e mulheres, entre ricos e pobres, urbano e rural, ou seja, é um colonialismo desterritorializado que produz exclusão social, patologização do cotidiano e medicalização da vida.

A descolonização é, portanto, um domínio crucial de luta, transformação do mundo e criação de outros modelos civilizatórios. Visa desmontar/desativar os dispositivos de poder, mitos, imaginários que estão na base dessa epistemologia dualista (mente/corpo; natureza/cultura; ocidente/resto) que defende o universo único. Os esforços de superação dessas formas colonialistas demandam novos quadros conceituais, novas concepções de subjetividade e suscita metodologias participativas, interventivas e emancipatórias. 
Busca-se produzir uma ontologia política que defende a vida em toda sua multiplicidade de formas e práticas, ancorada no relacional e comunitário, na diversidade e mutabilidade, incorporando diferentes cosmovisões, práticas culturais, etnias, gêneros, etc. Nesse paradigma, o mundo é um incessante e sempre cambiante fluxo de formas e de práticas. É uma multiplicidade. Mundo que comporta vários mundos. $\mathrm{E}$ alguns mundos não podem se impor sobre outros impossibilitando a sua existência (Escobar, 2014).

Tais aspectos impactam diretamente no modo como os profissionais procedem com as ações de acolhimento e organização da agenda para os casos de saúde mental, com desdobramento para a resolutividade das ações e serviços ofertados, levando em conta a realidade territorial e 0 contexto socioeconômico e cultural da população atendida. Por isso Guattari (1990) nos fala da exigência de considerar a articulação éticopolítica entre meio ambiente, relações sociais e subjetividade. Questiona por meio da Ecosofia, as maneiras de viver, as perspectivas tecnocráticas, as hegemonias de saber-poder, as serialidades, os racismos, - falocentrismo, indicando que as transformações deverão concernir não só às relações de forças visíveis, mas também aos domínios moleculares de sensibilidade, inteligência e desejo.

Este deve ser um princípio de primeira ordem na condução das relações e ações interprofissionais nas equipes e entre os serviços. $O$ apoio matricial, que se revelou como uma ferramenta pouco utilizada na qualificação clínica e suporte técnicopedagógico, poderia constituir-se como um dispositivo potente para que as equipes se desintoxicassem do discurso sedativo e saíssem da passividade fatalista, do dogmatismo insuportável, do empobrecimento das intervenções, e dos estereótipos que tornam os profissionais impermeáveis à alteridade singular tão recorrente nos serviços de saúde. Mas, para tanto, como nos lembra Guattari (1990), é necessário aderir à lógica das intensidades.

Parafraseando Basaglia (1979), diríamos que é preciso colocar a razão científica e tecnológica entre parênteses, a qual tem por base a negação da subjetividade e a serialização, produzindo uma objetivação extrema da pessoa como objeto do saber. Pessoas diferentes, com histórias, culturas, sofrimentos diferentes, entram na lógica universalizante dos vários campos de saber e vão passando por um processo de homogeneização, perdendo a originalidade de suas histórias, a espessura de seus sofrimentos. O resultado da avaliação do $P M A Q$, sob o recorte das questões que se relacionam direta ou indiretamente com a saúde mental, chama atenção para isso. Ou seja, que é preciso reverter essa operação e colocar os sujeitos em evidência, com suas diversidades, formas e projetos de vida.

À luz da ontologia política do território, de base relacional, a gestão integral do cuidado em saúde deve ocorrer a partir do conhecimento intenso das situações cotidianas, do território e das coletividades. É preciso potencializar o projeto de vida das comunidades baseado em suas práticas e cosmovisões, sair em defesa do território como espaço que sustenta esse projeto de vida desde a perspectiva étnico-territorial. $E$ oferecer possibilidades diversificadas de recompor outra corporeidade existencial, sair dos impasses repetitivos e da reterritorialização conservadora das subjetividades que empobrecem de modo contínuo nossa relação com 0 mundo (Guattari, 1990).

Assim, a questão colocada no início de como produzir uma 'nova institucionalidade' para o SUS e como articular as forças interessadas em enfrentar o reducionismo e empobrecimento do seu "devir existencializante", para nós tem uma direção: desmontando o colonialismo dos saberes, dos poderes e das subjetividades, criando uma textura ontológica heterogênea, nos 
ocupando dos sujeitos em suas experiências cotidianas, nos responsabilizando pela vida.

\section{REFERÊNCIAS}

Almeida, P. F., Santos, A. M., Santos, V. P., \& Silveira Filho, R. M. (2016). Integração assistencial em região de saúde: paradoxo entre necessidades regionais e interesses locais. Saude soc.,(25)2, 320-335. doi: 10.1590/S0104-12902016153295

Brasil (2010). Ministério da Saúde. Portaria GM/MS n. 4.279, de 30 de Dezembro de 2010. Estabelece diretrizes para a organização da Rede de Atenção à Saúde no âmbito do Sistema Único de Saúde (SUS). Brasília: Ministério da Saúde.

Brasil (2011a). Ministério da Saúde. Portaria № 3.088, de 23 de dezembro de 2011. Institui a Rede de Atenção Psicossocial para pessoas com sofrimento ou transtorno mental e com necessidades decorrentes do uso de crack, álcool e outras drogas, no âmbito do Sistema Único de Saúde (SUS) e dá outras providências. Brasília: Ministério da Saúde.

Brasil (2011b). Ministério da Saúde. Portaria GM no 1.654 de 19 de julho 2011. Institui, no âmbito do Sistema Único de Saúde, o Programa Nacional de Melhoria do Acesso e da Qualidade da Atenção Básica (PMAQ-AB) e 0 Incentivo Financeiro do PMAQ-AB, denominado Componente de Qualidade do Piso de Atenção Básica Variável PAB Variável. Diário Oficial da União, Brasília, DF.

Brasil (2015). Ministério da Saúde. SAS/DAPES. Coordenação Geral de Saúde Mental, Álcool e Outras Drogas. Saúde Mental em Dados - 12. Brasília. Recuperado de: http://www.saude.gov.br e http://www.saude.gov.br/bvs/saudemen tal
Brasil (2016). Ministério da Saúde. SAS/DAPES. Coordenação Geral de Saúde Mental, Álcool e Outras Drogas.

Saúde Mental no SUS: Cuidado em Liberda de, Defesa de Direitos e Rede de Atenç ão Psicossocial.

Relatório de Gestão 2011-2015.

Brasília. Recuperado de: http://u.saude.gov.br/images/pdf/2016/j unho/27/Relat--rio-Gest--o-2011-2015--.pdf

Basaglia, F. (1979). A psiquiatria alternativa: Contra o pessimismo da razão, o otimismo da prática. São Paulo: Brasil Debates.

Chiaverni, D. H. (Org.). (2011). Guia prático de matriciamento em saúde mental. Brasília, DF: Ministério da Saúde/ Centro de Estudo e Pesquisa em Saúde Coletiva.

Escobar, A. (2014). Sentipensar con la tierra: nuevas lecturas sobre desarrollo, territorio y diferencia. Medellin: Unaula.

Giovanella, L., \& Mendonça, M. H. M. (2008) Atenção Primária À Saúde. In: L.

Giovanella, S. Escorel, L. V. C. Lobato, J. C. Noronha, \& A. I. Carvalho (Orgs.), Políticas e sistema de saúde no Brasil (pp. 575-625). Rio de Janeiro: Fiocruz.

Guattari, F. (1990). As Três Ecologias. Campinas: Papirus.

Guattari, F. (1992). Caosmose: um novo paradigma estético. Rio de Janeiro: Editora 34.

Instituto Brasileiro de Geografia e Estatística. (2010). Censo Demográfico 2010: Características da população e dos domicílios. Resultados do universo. Rio de janeiro: IBGE.

Lima, J. G., Giovanella, L., Fausto, M. C. R., \& Bousquat, A. (2016). Qualidade da atenção básica por tipos de regiões de saúde. Novos Caminhos, N.12. Pesquisa Política,

Planejamento e Gestão das Regiões e Redes de Atenção à Saúde no Brasil. Recuperado de http://www.resbr.net.br/wpcontent/uploads/2016/07/NovosCaminh 012.pdf 
Lima, L. D. (2015). Condicionantes da regionalização da saúde no Brasil: desafios e recomendações para 0 planejamento e a gestão territorial do SUS no horizonte dos próximos 20 Anos. Rio de Janeiro: Fundação Oswaldo Cruz. Recuperado de http://saudeamanha.fiocruz.br/wpcontent/uploads/2016/07/15-

PJSSaudeAmanha_Texto0015_A4_2112-2015.pdf

Malta, D. C., Santos, M. A. S., Stopa, S. R., Vieira, J. E. B., Melo, E. A., \& Reis, A. A. C. D. (2016). A cobertura da Estratégia de Saúde da Família (ESF) no Brasil, segundo a Pesquisa Nacional de Saúde, 2013. Ciência \& Saúde Coletiva, 21(2), 327-338. doi: 10.1590/1413-81232015212.23602015

Ribeiro, P. T. (2015). Perspectiva territorial, regionalização e redes: uma abordagem à política de saúde da República Federativa do Brasil. Saúde e Sociedade, 24(2), 403-412. doi: 10.1590/S0104-12902015000200001

Santos, L., \& Campos, G. W. S. (2015). SUS Brasil: a região de saúde como caminho. Saúde e Sociedade, 24(2), 438-446. doi: $\quad 10.1590 / S 0104-$ 12902015000200004

Starfield, B. (2002). Atenção Primária: equilíbrio entre necessidades de saúde, serviços e tecnologia. Brasília: UNESCO, Ministério da Saúde.

Tavares, M. (2009). Epistemologias do Sul. Revista Lusófona de Educação, (13), 183-189. Recuperado de: http://www.scielo.mec.pt/scielo.php?scr ipt=sci_arttext\&pid=S1645$72502009000100012 \&$ lng=pt\&tlng=pt

Vasconcelos, E. M. (2010). Desafios políticos no campo da saúde mental na atual conjuntura: uma contribuição ao debate da IV Conferência Nacional. In: E. M. Vasconcelos (Org.), Desafios políticos da reforma psiquiátrica brasileira (pp. 17-74). São Paulo: Hucitec.

Viana, A. L. D., Bousquat, A., Pereira, A. P. C. M., Uchimura, L. Y. T., Albuquerque, M. V, Mota, P. H. S., Dermarzo, M. M.
P., \& Ferreira, M. P. (2015). Tipologia das Regiões de Saúde: condicionantes estruturais para a regionalização no Brasil. Saúde e Sociedade, 24(2), p.413-422. doi:10.1590/S010412902015000200002

\section{Nota de Rodapé}

1. http://www.ensp.fiocruz.br/portalensp/informe/site/materia/detalhe/41156 